\title{
Requirements Quality Online Courses
}

\author{
Dragan Damjanović \\ Master of Science, Zrenjanin, Serbia \\ Email: damjanovic1971@gmail.com
}

Received 16 January 2014; revised 15 February 2014; accepted 13 March 2014

Copyright (C) 2014 by author and Scientific Research Publishing Inc.

This work is licensed under the Creative Commons Attribution International License (CC BY).

http://creativecommons.org/licenses/by/4.0/

(c) (i)

Open Access

\begin{abstract}
Every day on the Internet appear online courses (good, bad, free, expensive, legal, illegal, ...). They can be of great benefit, especially to people who, because of a lack of free time can not attend face-to-face teaching, and have a duty of continuous professional development. In this great offer, it is really difficult to choose the right one that will fully meet the strict criteria. In this paper, we point to the conditions and requirements that may significantly facilitate students making their final decision regarding the selection of different forms of online training.
\end{abstract}

\section{Keywords}

\section{Courses, Online, Quality, Requirements}

\section{Introduction}

Suffice it to connect to the internet and in the short time that I have lavished on advertising on various online courses from abroad to domestic. Whenever I see them, I think that you can just fill out a registration form, enter your information and sign up for some of them in a matter of days that you will receive a certificate. Certificate of having passed the course will of course recognize all. Therefore, I decided to go a little further to analyze this topic. First, I found that a large number of platforms that allow authors' courses quickly and easily (sometimes free, sometimes not) to create and deploy courses. As everything in this area is dominated by a few companies, the original solutions are no longer on the market, with most of its clients, who collaborate with the most eminent universities around the world, and the career's prescribed rules and establish standards are to be met by online courses, because of this issue very seriously. The hypothesis of this paper will be set so that it can help all potential users and online courses help students choose the best possible quality of course, that is to recognize those who truly meet the necessary standards. Hypothesis: standard courses are an important factor in raising the quality of online courses, without respect that there is no quality standards and a recognized course. That means anyone who wants to attend the course should keep in mind that they will not succeed if you expand your knowledge of the chosen course which does not meet basic standards that will be discussed in this paper. 


\section{Course Design}

Course Design addresses elements of instructional design. For the purpose of this program, course design includes such elements as structure of the course, learning objectives, organization of content, and instructional strategies.

\subsection{Goals and Objectives}

Exemplary: Goals and objectives are easily located within the course; Goals and objectives are clearly written at the appropriate level and reflect desired outcomes; Goals and objectives are written in measureable outcomes (students know what they are expected to be able to do); Goals and objectives are made available in a variety of areas in the course (within the syllabus and each individual learning unit).

Accomplished: Goals and objectives are located within the course syllabus or the individual learning units; Objectives are written to reflect desired learning outcomes, although not all are written as measureable outcomes; Students have some understandings of what is expected of them.

Promising: Goals and objectives are not easily located within the course; Goals and objective are not clearly written in measurable learning outcomes; Students may be unsure of what they are expected to be able to do; The level does not match the desired outcomes.

Incomplete: Goals and objectives are not easily located within the course; Some are missing and others poorly written; The level does not match the desired learning outcomes.

\subsection{Content Presentation}

Exemplary: Content is made available or "chunked" in manageable segments (i.e., presented in distinct learning units or modules); Navigation is intuitive; Content flows in a logical progression; Content is presented using a variety of appropriate mechanisms (content modules, single pages, links to external resources,); Content is enhanced with visual and auditory elements; Supplementary resources are made available and are wellintegrated with other course materials (integrated publisher resources, e-textbooks, course manuals, etc.).

Accomplished: Content is made available or "chunked" in manageable segments (i.e., presented in distinct learning units or modules); Navigation is somewhat intuitive, but some "exploring" is required to determine the flow of content; Content is presented using a variety of mechanisms (content modules, single pages, links to external resources, RSS Feeds, print material); Visual and/or auditory elements occasionally enhance the content; supplementary resources are made available (course CDs, textbooks, course manuals, etc.).

Promising: Some content segments are overly large (or possibly too small) for the specified objectives; Navigation is only occasionally intuitive, thus the flow of content is sometimes not easily determined; The design does not avail of the content presentation tools (content modules, single pages, links); Few or no visual and/or auditory elements are used to enhance the content; Supplementary resources may be made available (course CDs, textbooks, course manuals, etc.).

Incomplete: Content is not "chunked" into manageable segments; Navigation is not intuitive and the flow of content is unclear; The design does not avail of the content presentation tools (content modules, single pages, links); No visual or auditory elements are used to enhance the content; Supplementary resources are not made available (course CDs, textbooks, course manuals, etc.).

\subsection{Learner Engagement}

Exemplary: It is clear how the instructional strategies will enable students to reach course goals and objectives; Course design includes guidance for learners to work with content in meaningful ways; Higher order thinking (e.g., analysis, problem solving, or critical reflection) is expected of learners and explained with examples or models; Individualized instruction, remedial activities, or resources for advanced learning activities, such as integrated publisher resources, are provided.

Accomplished: Instructional strategies are designed to help students reach course goals and objectives, although this relationship may not be obvious to learners; Guidance is provided, but could be improved with greater detail or depth higher order thinking is required for some activities but is not well-explained or supported (e.g., by providing examples of "good answers"); Differentiated instruction (such as remediation) may be available on a limited basis. 
Promising: It is not clear how the instructional strategies will help learners achieve course goals and objectives; Guidance in using content materials may only be provided on a limited basis; Higher order thinking is not required or encouraged; Differentiated instructional opportunities are not provided, although there may be supplementary content resources available.

Incomplete: Instructional strategies do not provide students with skills needed to achieve course goals and objectives; Content is provided but it is not clear what students are expected to do with it; Higher order thinking is not expected from students No supplementary resources or activities are provided for remediation or advanced study.

\subsection{Technology Use}

Exemplary: Tools available within the LMS are used to facilitate learning by engaging students with course content; LMS tools are used to reduce the labor-intensity of learning (e.g., providing links to needed resources which they will be used in the course, integrating publisher resources that are tailored to the course materials, and providing streamlined access to supplementary materials); Technologies are used creatively in ways that transcend traditional, teacher-centered instruction A wide variety of delivery media are incorporated into the course.

Accomplished: Tools available within the LMS could be utilized more (or more creatively) to engage learners with course content; LMS tools are made available to assist students, but could be organized or arranged for even greater usefulness; Technologies within the course are used in many cases merely to replicate traditional face-to-face instruction There is some variety in the tools used to deliver instruction.

Promising: Tools available within the LMS are not used to their full extent or not used when it would be appropriate to do so; Only a few tools (of those available within the LMS) are used in a way that streamlines access to materials and activities for students. Technologies within the LMS are used primarily by instructors and not students ("students as recipients of content" model); There is little variety in use of technologies within the LMS.

Incomplete: Technologies used within the LMS do not engage students with learning; Tools that could reduce the laborintensity of online instruction are not utilized; Students are not expected to use technologies available within the LMS; Only a few technologies available within the LMS are used.

\section{Interaction and Collaboration}

Interaction and Collaboration can take many forms. The ECP criteria place emphasis on the type and amount of interaction and collaboration within an online environment. Interaction denotes communication between and among learners and instructors, synchronously or asynchronously. Collaboration is a subset of interaction and refers specifically to those activities in which groups are working interdependently toward a shared result. This differs from group activities that can be completed by students working independently of one another and then combining the results, much as one would when assembling a jigsaw puzzle with parts of the puzzle worked out separately then assembled together. A learning community is defined here as the sense of belonging to a group, rather than each student perceiving himself/herself studying independently [1].

\subsection{Communication Strategies}

Exemplary: There are plentiful opportunities for synchronous and/or asynchronous interaction, as appropriate; Asynchronous communication strategies promote critical reflection or other higher order thinking aligned with learning objectives; Synchronous communication activities benefit from real-time interactions and facilitate "rapid response" communication (i.e., students gain practice discussing course content extemporaneously without looking up basic, declarative information).

Accomplished: Several communication activities are included to reinforce the desired learning outcomes; Asynchronous communications sometimes require reflection or other higher order thinking; Synchronous interactions are meaningful but may not take full advantage of the realtime presence of instructor and/or peers.

Promising: Communication strategies are included, however, they may not consistently reinforce desired learning outcomes; Asynchronous communications are focused primarily on lower levels of thinking (e.g., summarizing, describing, interpreting, etc.); Synchronous interactions are used mostly for instructor explanation 
or clarification of content, or other instructor-focused activities.

Incomplete: Little to no attention has been devoted to communication strategies; Interaction activities that are included do not invoke critical thinking, reinforce learning, or take advantage of the specific strengths of the communication tools used.

\subsection{Development of Learning Community}

Exemplary: Communication activities are designed to help build a sense of community among learners; Student-to-student interactions are required as part of the course; Students are encouraged to initiate communication with the instructor; Collaboration activities (if included) reinforce course content and learning outcomes, while building workplace-useful skills such as teamwork, cooperation, negotiation, and consensus-building.

Accomplished: Communication activities may help learners build a sense of community, but do not appear to be designed with this in mind; Some student-to-student interaction is built into the course; Students interact with the instructor, although primarily as a result of instructorinitiated contact; Collaboration activities (if included) support some team-building skills, but may not purposefully integrate these elements.

Promising: Effort has been devoted to fostering a sense of community in the course, but only minimally; More focus is needed on designing activities and a course climate that foster student-to-student interactions as well as student-to-instructor interactions.

Incomplete: Little to no attention has been devoted to building a sense of community in this course.

\subsection{Interaction Logistics}

Exemplary: Guidelines explaining required levels of participation (i.e., quantity of interactions) are provided; Expectations regarding the quality of communications (e.g., what constitutes a "good" answer) are clearly defined; A rubric or equivalent grading document is included to explain how participation will be evaluated; The instructor actively participates in communication activities, including providing feedback to students; The instructor uses communication tools to provide course updates, reminders, special announcements, etc.

Accomplished: Expectations of student participation in communication activities are given, but would benefit from more detail; Expectations regarding the quality of communications are included, but may be sketchy and lack detail or illustrative examples; Minimal information may be provided regarding grading criteria for communications activities; The instructor is occasionally involved in communication activities; The instructor sometimes takes advantage of LMS tools to post announcements, reminders, etc.

Promising: Instructor expectations of student interactions are not made clear; Little information is provided regarding what constitutes a "good” response or posting; Students are not given a clear set of criteria for how communications activities will be graded; The instructor appears to be largely absent from communication activities.

Incomplete: Few announcements, reminders, or other updates are provided; Few or no guidelines are provided to students regarding the desired quantity or quality of communications/interactions within the course; The instructor does not participate in communications activities with students.

\section{Assessment}

Assessment focuses on instructional activities designed to measure progress towards learning outcomes, provide feedback to students and instructor, and/or enable grade assignment. This section addresses the quality and type of student assessments within the course [2].

\subsection{Expectations}

Exemplary: Assessments match the goals \& objectives; Learners are directed to the appropriate objective(s) for each assessment; Rubrics or descriptive criteria for desired outcomes are provided (models of "good work" may be shown, for example); Instructions are written clearly and with sufficient detail to ensure understanding.

Accomplished: Assessments match the goals \& objectives; Rubrics or descriptive criteria for desired outcomes are included for some assessment activities; Instructions are written clearly, with some detail included.

Promising: Students are assessed on the topics described in the course goals and objectives; There may be some explanation of how assessments will be scored/ graded; Instructions lack detail that would help students 
understand how to complete the activities.

Incomplete: Assessments bear little resemblance to goals \& objectives; Expectations or grading criteria are not provided; Instructions are limited or absent.

\subsection{Assessment Design}

Exemplary: Assessments appear to measure the performance they claim to measure (e.g., activities are explained using appropriate reading level and vocabulary); Higher order thinking is required (e.g., analysis, problem-solving, etc.); Assessments are designed to mimic authentic environments to facilitate transfer; Assessment activities occur frequently throughout the duration of the course; Multiple types of assessments are used (research project, objective test, discussions, etc.).

Accomplished: Assessment activities have "face validity" (i.e., they appear to match the curriculum); Some activities involve higher order thinking; Assessment activities may focus on tasks similar to real-world application of skills; Multiple assessments are included; at least three different types of assessments are used.

Promising: It is not clear whether the assessment activities actually measure the desired skill; The vast majority ofassessments require only low-level thinking (memorization, for example); Assessment activities typically do not include tasks that are relevant beyond the scope of this course; multiple assessments are included; Two types of assessments are included, at a minimum.

Incomplete: Assessment activities appear to lack validity due to bias, lack of clarity in questions or tasks, or because students are evaluated on performance unrelated to the stated objectives; No higher-order thinking skills are required to complete assessment activities; There is little or no evidence of authenticity built into assessments; Assessments are too few and far apart for the course content.

\subsection{Self-Assessment}

Exemplary: Many opportunities for self-assessment are provided; Self-assessments provide constructive, meaningful feedback.

Accomplished: Some self-assessment activities are included; Self-assessments provide feedback to learners.

Promising: There may be selfassessment activities, but they are limited in scope and do not offer useful feedback.

Incomplete: A few self-assessments may be included, but they offer little more feedback than flash cards.

\section{Learner Support}

Learner Support addresses the support resources made available to students taking the course. Such resources may be accessible within or external to the course environment. Specifically, learner support resources address a variety of student services [3].

\subsection{Orientation to Course and LMS}

Exemplary: Clearly labeled tutorial materials that explain how to navigate the LMS and the specific course are included; Tutorials are found easily (few clicks) whether internal or external to the course, with easy return to other areas of the course; Tutorial materials support multiple learning modalities: audio, visual, and text based.

Accomplished: Clearly labeled tutorial materials that explain how to navigate the LMS and the specific course are included; Tutorials may not be easily accessed, or require the learner to leave course site without an easy return; Tutorial materials support multiple learning modalities: audio, visual, and text based.

Promising: Tutorial materials that explain how to navigate the LMS and/or the specific course may be evident, but not easily found; Materials do not support multiple learning modalities and are textbased only.

Incomplete: Tutorial materials explaining how to navigate the LMS or the specific course may be included but are difficult to find, lack detail, are not well organized, or are incomplete; Tutorial materials that are included do not support learning modalities.

\subsection{Supportive Software (Plug-Ins)}

Exemplary: Clear explanations of optional and/or required software including any additional costs are 
provided within the course; Software required to use course materials is listed with links to where it can be captured and installed; Links are located within the course where learners will use the software (i.e., near the materials requiring its use).

Accomplished: Clear explanations of optional and/or required software (in addition to the LMS) are provided within the course; Software required to use course materials is listed but links to where it can be captured and installed are not found near where it will be used.

Promising: Software (in addition to the LMS) required to use course materials is mentioned, but not explained; Links to where it can be captured and installed are provided, although they may not be conveniently located.

Incomplete: The need for additional software required to use course materials may be mentioned; Links to software may be missing or incomplete.

\subsection{Instructor Role and Information}

Exemplary: Contact information for the instructor is easy to find and includes multiple forms of communication (for example, e-mail, phone, chat, etc.); Expected response time for e-mail replies is included; The instructor's role within the course is explained (for example, whether he/she will respond to "tech support" type questions); The instructor's methods of collecting and returning work are clearly explained.

Accomplished: Contact information for the instructor is included but may not be easy to find; contact information includes more than one type of communication tool; Expected response time for e-mail replies may be included; Instructor's role within the course is not clearly spelled out to students; The instructor's methods of collecting and returning work are clearly explained.

Promising: Contact information for the instructor is provided but not easy to find; Contact information includes only one way to reach the instructor; Information concerning response time for e-mail replies is not included; Little or no information is given regarding the instructor's role in the course; The instructor's methods of collecting and returning work are evident but not clearly explained.

Incomplete: Contact information for the instructor is sketchy, at best; Lacks information concerning response time for e-mail replies is included; Information regarding the instructor's role in the course is not included; Instructor's methods of collecting and returning work are confusing or non-existent.

\subsection{Course/Institutional Policies \& Support}

Exemplary: Links to institutional policies, materials, and forms relevant for learner success (for example, plagiarism policies) are clearly labeled and easy to find; Links allow easy navigation from the course to the information and back; course/instructor policies regarding decorum, behavior, and netiquette are easy to find and written clearly to avoid confusion; Links to institutional services such as the library, or writing center, are clearly labeled and easy to find.

Accomplished: Links to institutional policies, materials, and forms relevant for learner success (for example, plagiarism policies) are included but may require searching to find; Links allow easy navigation from the course to the information and back; Course/instructor policies regarding decorum, behavior, and netiquette are included and are written clearly to avoid confusion; Links to institutional services such as the library, writing center, or financial aid office may be included but require searching to find.

Promising: Links to some institutional policies, materials, and forms relevant for learner success (for example, plagiarism policies) are included but are difficult to find; Course/instructor policies regarding decorum, behavior, and netiquette are included but are not clearly written or would benefit from more detail; A few links to institutional services such as the library, writing center, or financial aid office may be included but require searching to find.

Incomplete: Links to some institutional policies, materials, and forms relevant for learner success (for example, plagiarism policies) are not included; Some course/instructor policies regarding decorum, behavior, and netiquette may be included but are not clearly written or would benefit from more detail; Links to institutional services such as the library, writing center, or financial aid office are not included.

\subsection{Technical Accessibility Issues}

Exemplary: Course materials use standard formats to ensure accessibility; If specific software is required to 
which some learners may not have access, alternative file types are provided; Large files are identified to help learners consider download times; Alternative (smaller) files are provided where appropriate; Videos are streamed whenever possible; graphics are optimized for web delivery and display without needing extensive scrolling.

Accomplished: Course materials use standard formats to ensure accessibility; If specific software is required to which some learners may not have access, alternative file types are sometimes provided; Large files are not identified as such alternative (smaller) files are not provided; Video files are streamed in some cases; Graphics are not be optimized for web delivery but display without extensive scrolling.

Promising: Course materials use standard formats to ensure accessibility; If specific software is required to which some learners may not have access, alternative file types are not provided; Large files are not identified as such and alternative (smaller) files are not provided Video files are not streamed; Graphics are not optimized for web delivery and may require extensive scrolling.

Incomplete: Course materials sometimes use standard formats to ensure accessibility; If specific software is required to access course materials, no mention of this is included and alternative file types are not provided; Large files are not identified as such and alternative (smaller) files are not provided; Video files are not streamed; Graphic files are not optimized for web delivery and require extensive scrolling.

\subsection{Accommodations for Disabilities}

Exemplary: Supportive mechanisms allow learners with disabilities to participate fully in the online community; The design and delivery of content integrate alternative resources (transcripts, for example) or enable assistive processes (voice recognition, for example) for those needing accommodation; Links to institutional policies, contacts, and procedures for supporting learners with disabilities are included and easy to find; Design factors such as color, text size manipulations, audio and video controls, and alt tags reflect universal accessibility considerations.

Accomplished: Supportive mechanisms allow learners with disabilities to participate in the online community for most activities; The design and delivery of content integrate some alternative resources or enable assistive processes for those needing accommodation; Links to institutional policies, contacts, and procedures to support learners with disabilities are included but may not be easy to find; Design factors such as color, text size manipulation, audio and video controls, and alt tags have been considered in some cases.

Promising: Supportive mechanisms allow some learners with disabilities to participate fully in the online community; The design and delivery of content do not include alternative resources nor enable assistive processes for those needing accommodation; Links to institutional policies, contacts, and procedures to support learners with disabilities are not evident; Design factors such as color, text size manipulation, audio and video controls, and alt tags have not been considered.

Incomplete: Supportive mechanisms allow some learners with disabilities to participate in the online community for some activities; The design and delivery of content do not apply alternative resources nor enable assistive processes for those needing accommodations; Links to institutional policies, contacts, and procedures to support learners with disabilities are not evident; Design factors such as color, text size manipulation, audio and video controls, and alt tags have not been considered.

\subsection{Feedback}

Exemplary: Learners have the opportunity to give feedback to the instructor regarding course design and course content both during course delivery and after course completion; Feedback mechanisms allow students to participate anonymously in course evaluation.

Accomplished: Learners have the opportunity to give feedback to the instructor regarding course design and/or course content, but only after course completion; Feedback mechanisms allow students to participate anonymously in course evaluation.

Promising: Learners have the opportunity to give feedback to the instructor regarding course design or course content, but only after course completion; Feedback mechanisms do not guarantee privacy to the student.

Incomplete: Learners do not have the opportunity to give feedback to the instructor regarding course design or course content; Feedback mechanisms do not guarantee privacy to the student. 


\section{Conclusion}

All this leads to the conclusion that it is not easy to choose a course. It is certain that the work hypothesis is confirmed, and a conclusion on this issue is that without respect of these standards, there is no quality online courses, because studies show that they are better students who attended a standardized training courses. This would especially be of great value to teachers, professors, educators, with a duty of continuous professional development, because the changes in the field of their work commitments often occur, and they need to be in constant touch with the latest developments, hence the development of even more necessary. However, this does not mean that it should be abused, so these courses and seminars charging schools and educators at enormously high prices mostly would be even supposed to be free. Because the platform used for creating online courses and seminars are free of charge, which can be found on the internet. This, however, could be the subject of a new study.

\section{References}

[1] Cavanaugh, C. (2009) Virtual Schooling Standards and Best Practices for Teacher Education. Journal of Technology and Teacher Education, 17, 1059-7069.

[2] Patrick, S. and Powell, A. (2009) A Summary of Research on the Effectiveness of K-12 Online Learning. International Association for K-12 Online Learning.

[3] The National Council for Higher Education (2009) Instructions for the Preparation of Documents for the Accreditation of Degree Programs at a Distance. 